\title{
A Vision for Platform Security
}

\author{
Ernie Brickell \\ Intel, United States \\ ernie.brickell@intel.com
}

Intel has recently produced several new capabilities to enhance security on the platform that have been released or will be released in the near future. In this presentation I will give a review of these capabilities and discuss their benefit to the security of the platform. 